\section{SUPRACLAVICULAR BLOCK FOR ELBOW FRACTURES}

W Kamal*. Elyoussef Medical Teaching Hospital, Military Central Hospital, Akkar, Lebanon

\subsection{6/rapm-2021-ESRA.23}

Background and Aims The aim is to demonstrate the efficiency of supraclavicular block with a mixture of Bupivicaine $0.5 \%$ and lidocaine $2 \%$.

Methods Patients with elbow fractures were scheduled for supraclavicular block using a mixture (50/50) of bupivicaine $0.5 \%$ and lidocaine $2 \%$. The volume used was $40 \mathrm{ml}$. Dexamethasone $8 \mathrm{mg}$ was added i. V. It was opened surgery with insertion of 2 plaques with screws. Duration of operations was 2-3 hours. Some patients who were put in sitting position received an intubation with $50 \mathrm{mcg}$ fentanyl, propofol $150 \mathrm{mg}$, rocuronium $30 \mathrm{mg}$, and the maintenance was on sevoflurane $1 \%$, with oxygene/air(50/50). Patients that were put laterally received midazolam $1-3 \mathrm{mg}$ iv, snd $50-70 \mathrm{mg}$ propofol and maintained on spontaneous respiration on oxygen mask.

Results Perioperatively, the pulse, heart rate and pressure did not change more then $20 \%$. Patients were stable hemodynamically. They were sent to the recovery room to be observed for $30 \mathrm{~min}$ and tben transferred to the wards. They received paracetamol $1 \mathrm{~g}$ iv/6h and ketorolac $30 \mathrm{mg}$ iv/8h. The EVA was always $<4$. They went home on the next day.

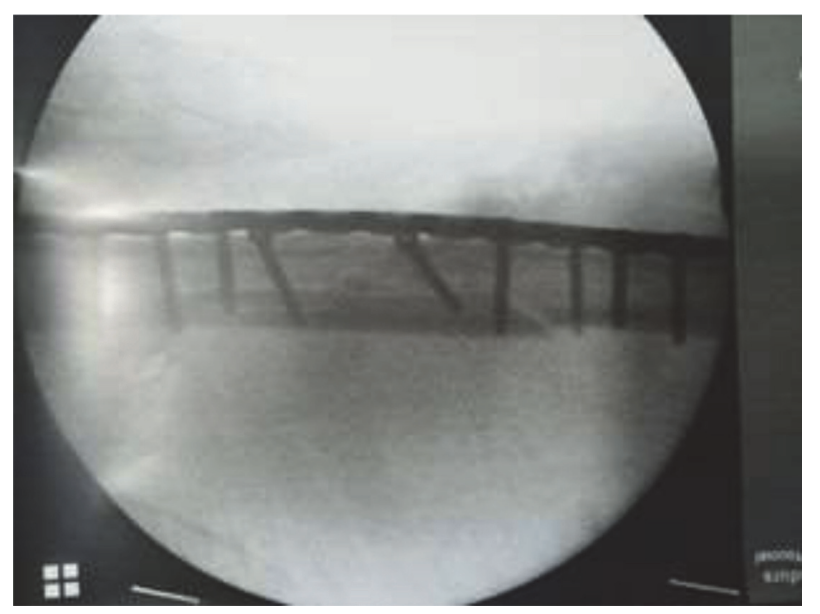

Abstract 23 Figure 1

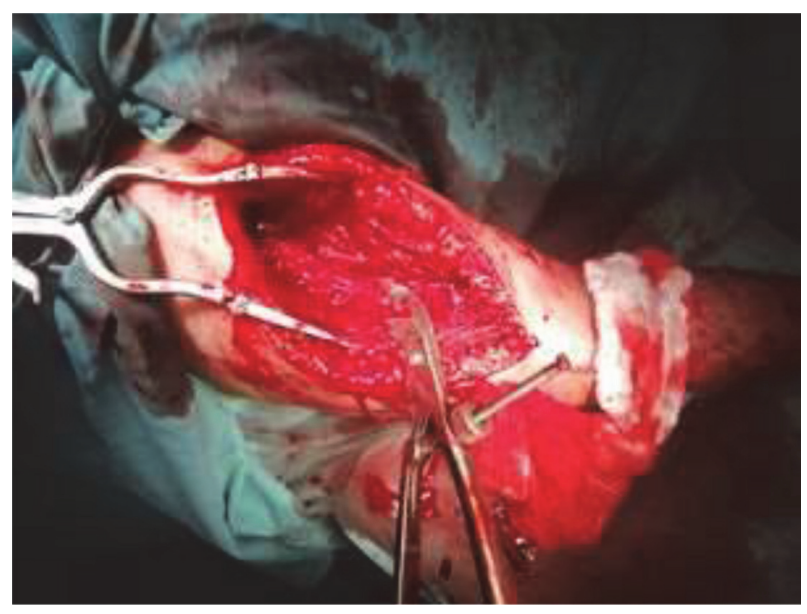

Abstract 23 Figure 2

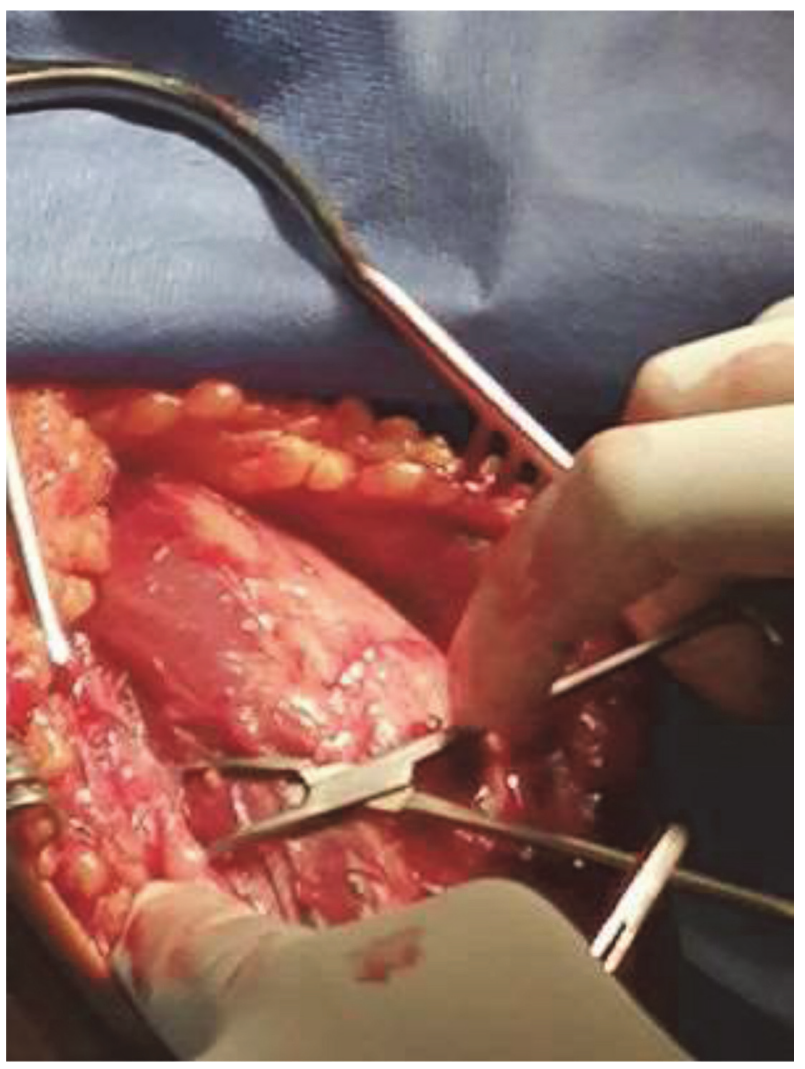

Abstract 23 Figure 3

Conclusions A mixture of bupivicaine $0.5 \%$ isobar with lidocaine $2 \%$, with a total volume of $40 \mathrm{ml}$ and dexamethasone $8 \mathrm{mg}$ iv, gives adequate analgesia and anesthesia for elbows fractures that were operated by insertion of plaques.

\section{ANTERIOR CERVICAL EPIDURAL HEMATOMA AFTER COMBINED SPINAL EPIDURAL ANESTHESIA: A CASE REPORT}

SK Coşarcan* , AT Doğan, D Akbay, Ö Erçelen. VKV American Hospital, Istanbul, Turkey

\subsection{6/rapm-2021-ESRA.24}

Background and Aims The choice of anesthetic technique should be based on the maternal and fetal condition, comorbidities, the expected duration and difficulty of the procedure, and the presence or absence of an in-situ epidural or spinal catheter. Venous thromboembolism (VTE) remains an important cause of morbidity and mortality in the obstetric population. This article presents a case about cervical anterior epidural bleeding and conservative management seen after cesarean with combined spinal-epidural anesthesia.

Methods A 32-year-old woman was admitted to our clinic for a cesarean operation for 36 weeks twin pregnancy. A combined spinal-epidural was planned for the cesarean operation anesthesia. After the operation, the patient with no complaints was transferred to the inpatient clinic by adjusting the epidural patient-controlled analgesia device (PCA) doses.

Results She had a headache in the posterior region of the head that spreads neck and shoulders started 48 hours after the operation. The next day patient was pain-free with analgesic medications and has discharged with low molecular weight 
heparin prophylaxis. On the postoperative 6th day, neck and shoulder pain were worsened unless she took any anti-inflammatory drug. In the cervical and thoracic MRI, there was a hematoma was detected. Intravenous hydration and antiinflammatory therapy were organized. The neck and shoulder pain on the second day of his second admission decreased dramatically.

Conclusions The most important thing to mention is that PDDH can mask the hematoma-induced headache. It should be kept in mind that a spinal hematoma can be observed even VTE prophylaxis was started according to guidelines.

\section{CONTINUOUS SPINAL ANESTHESIA AS A SAFE ALTERNATIVE FOR RETROPUBIC PROSTATECTOMY IN A HIGH-RISK PATIENT}

P Rebelo, M Cunha, C Pereira*. Hospital Pedro Hispano, Matosinhos, Portugal

\subsection{6/rapm-2021-ESRA.25}

Background and Aims Anesthetic management of patients with pulmonary hypertension $(\mathrm{PH})$ is complex and carries a high potential of perioperative morbidity and mortality.

Methods We report a continuous spinal anesthesia (CSA) for retropubic prostatectomy.

Results A 71-year-old man, ASA IV with a very severe COPD conditioning severe $\mathrm{PH}$ was proposed for retropubic prostatectomy. Because of the risks of general anesthesia (GA) in this patient, we decided to perform a CSA with the IntraLong kit from PAJUNK. In lateral position under full aseptic precaution, a $21 \mathrm{G}$ pencil point needle was inserted into the L3-L4 space. After obtaining free flow of cerebrospinal fluid, a $25 \mathrm{G}$ catheter was introduced. We started with $5 \mathrm{mg}$ of levobupivacaine. With a light slope the dose was titrated $(2,5 \mathrm{mg}$ at the time) until adequate block was achieved. During surgery, the patient was always hemodynamically stable and we used a total of $15 \mathrm{mg}$ of levobupivacaine.

At the end of the surgery the catheter was withdrawn, and postoperative recovery was uneventful.

Conclusions The anesthetic management of a patient with $\mathrm{PH}$ is a challenge. GA with positive pressure ventilation in associated with a potential risk of cardiorespiratory decompensation. However, spinal anesthesia is associated with a risk of severe hypotension which can be catastrophic in a patient with low functional capacity. With the use of CSA we can titrate the local anesthetic to maintain hemodynamic stability in the perioperative period with no vasopressors needed and with spontaneous ventilation with a peripheral saturation $>92 \%$.

\section{ANAESTHETIC CONSIDERATIONS: A CASE OF MCCUNE- ALBRIGHT SYNDROME POSTED FOR OPTIC NERVE DECOMPRESSION - A CASE REPORT}

${ }^{1} \mathrm{R}$ Kashyap, ${ }^{2} \mathrm{~K}$ R*, ${ }^{2} \mathrm{D}$ Goswami. ${ }^{1}$ Chandra Laxmi Hospital, Vaishali, India; ${ }^{2}$ All India Institute of Medical Sciences, New Delhi, India

\subsection{6/rapm-2021-ESRA.26}

Background and Aims Mccune Albright syndrome (MAS) consists of a triad of Fibrous dysplasia, café-au-lait macules and autonomous hyperfunctoning endocrinopathies, caused by mutation in $G_{s} \alpha$ subunit of G-protein coupled receptors (GPCRs). Anaesthetic management of these patients could be complicated by difficult airway, increased risk of perioperative arrhythmias, other manifestations of associated endocrinopathies and pathological fractures.

Methods We present the anaesthetic management of a 13 years old boy, case of MAS posted for optic nerve decompression. He had history of multiple fractures involving upper and lower limbs. Drug history included tab carbimazole for hyperthyroidism, calcitriol, calcium and sodium acid phosphate for dysplastic bone. Airway examination revealed coarse facial features with large head and tongue with restricted neck movement towards the right side. NCCT orbit revealed narrowed optic canal secondary to expanded dysplastic bone, causing vision loss in right eye. On the day of surgery, radial artery cannulation was done under local anaesthesia. Intravenous induction was done with fentanyl, propofol and atracurium along with dexmedetomidine infusion. C-Mac video laryngoscope and bougie guided intubation done.

Results Anaesthesia was maintained with oxygen, nitrous oxide, isoflurane, atracurium boluses and dexmedetomidine infusion. Patient positioning was done with extreme care. Paracetamol, ketorolac and morphine were given for pain control. Surgery was uneventful. The patient was shifted to ICU for postoperative monitoring and extubated the next day.

Conclusions Throughout surgery, the patient had increased requirement of anaesthetic agents. GPCRs have been considered as potential targets of inhalational anaesthetics, whether this could have an implication on patients with MAS needs further research.

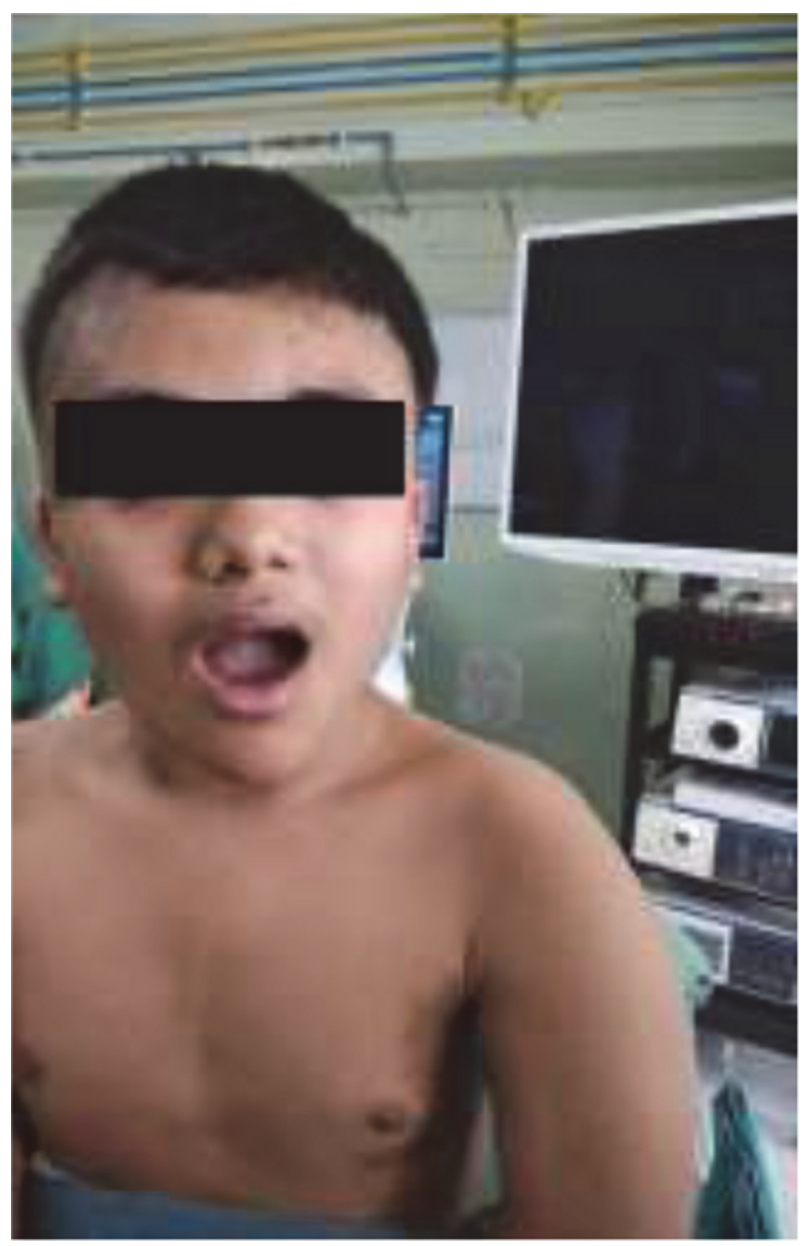

Abstract 26 Figure 1 